\title{
Mediastinal lymphadenectomy under laparoscopic assisted single-port inflatable mediastinoscopy through left neck approach
}

\author{
Xiangfeng Gan ${ }^{1,2 \#}$, Hongcheng Zhong ${ }^{1,2 \#}$, Xiaojian Li $^{1}$, Xiaojin Wang ${ }^{1}$, Wenwen Huo ${ }^{1}$, Tianchi Wu ${ }^{1}$, \\ Beilong Zhong ${ }^{1}$, Bin Zhang ${ }^{1}$, Hua Cheng ${ }^{1}$, Xiangwen Wu ${ }^{1,2}$, Qingdong Cao ${ }^{1,2}$ \\ ${ }^{1}$ Department of Cardiothoracic Surgery, ${ }^{2}$ Guangdong Provincial Key Laboratory of Biomedical Imaging, the Fifth Affiliated Hospital of Sun Yat-sen \\ University, Zhuhai, China \\ \#These authors contributed equally to this work. \\ Correspondence to: Qingdong Cao; Xiangwen Wu. Department of Thoracic Surgery, the Fifth Affiliated Hospital of Sun Yat-sen University, No. 52 \\ East Meihua Road, Xiangzhou District, Zhuhai 519000, China. Email: foryours.gan@msn.com; wxwcpums@163.com.
}

\begin{abstract}
Minimally invasive esophagectomy (MIE) has been developed for decades. However, conventional MIE requires transthoracic surgery, which can increase the risk of many perioperative cardiopulmonary complications. Therefore, mediastinoscopy-assisted transhiatal esophagectomy has been proposed, but the traditional surgical methods have shortcomings, such as unclear vision, especially during the dissection of mediastinal lymph nodes (LNs). A new approach for mediastinal lymphadenectomy under single-port inflatable mediastinoscopy with one left-neck incision is proposed. There are three difficulties in this procedure. (I) LNs along the left recurrent laryngeal nerve (RLN). After establishing pneumomediastinum, esophagectomy is performed over the aortic arch to the level of the lower edge of the left main bronchus, and lymphadenectomy along the left RLN is also accomplished during this process. (II) LNs along the right RLN. At the level of the lower edge of the right subclavian artery (RSA), between the trachea and the esophagus, instruments are used to access the right RLN. Lymphadenectomy of up to $2 \mathrm{~cm}$ can be accomplished at the upper edge of the RSA. (III) Subcarinal LNs. Between the trachea and esophagus, the left and right main bronchi are exposed along the trailing edge of the trachea down to the carina, and lymphadenectomy can be performed here. The surgical procedure described here in detail is the first mediastinal lymphadenectomy under mediastinoscopy with one single left-neck incision.
\end{abstract}

Keywords: Lymphadenectomy; mediastinoscopy; minimally invasive esophagectomy (MIE); lymph nodes (LNs)

Submitted Jan 10, 2020. Accepted for publication Jul 03, 2020.

doi: $10.21037 /$ tcr-20-467

View this article at: http://dx.doi.org/10.21037/tcr-20-467

\section{Introduction}

Esophageal cancer ranks 9th in terms of the incidence of malignant tumors worldwide and 6th in terms of the mortality rate (1); in China, esophageal cancer ranks 6th and 4th (2), showing that China faces a higher burden from esophageal cancer when measured against the global average. Minimally invasive esophagectomy (MIE) has been developed for decades. However, conventional MIE requires transthoracic surgery, which can increase the risk of many perioperative cardiopulmonary complications (3). Therefore, mediastinoscopy-assisted transhiatal esophagectomy has been proposed, but the traditional surgical methods have shortcomings, such as unclear vision, especially during the dissection of mediastinal lymph nodes (LNs).

To this end, Professor Fujiwara proposed a new mediastinoscopic esophagectomy in 2015 (4). In addition to non-transthoracic esophagectomy, this new surgery also performed lymphadenectomy along the bilateral recurrent laryngeal nerves (RLNs) through two neck incisions. This procedure involves a left neck incision to dissect the esophagus and remove LNs along the left RLN. A second incision is made at the anterior edge of the 

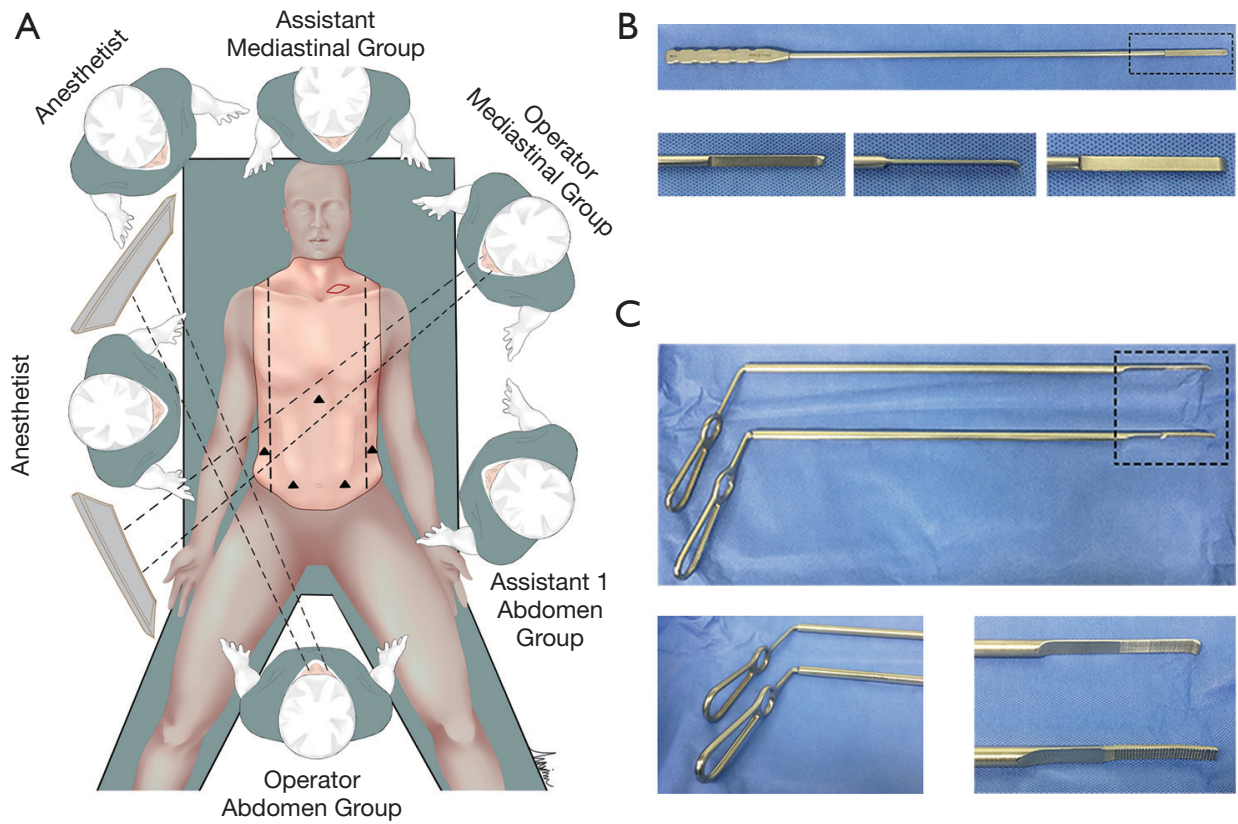

Figure 1 General preparation of the procedure. (A) The position of surgeons and assistants. (B) The upper mediastinal retractor (5-mm width and 35 -cm length). (C) The lower mediastinal retractor (10-mm width and 45-cm length).

sternocleidomastoid muscle on the right side of the neck for lymphadenectomy. To reduce trauma and avoid double incision of the neck, we proposed a new surgical approach for dissecting LNs along the bilateral RLNs with a single left neck approach. This procedure avoids a right neck incision, and it is possible to perform lymphadenectomy along the bilateral RLNs and subcarinal through a left neck incision.

\section{Surgical process}

This novel surgical procedure was performed on 56 ESCC patients at the Fifth Affiliated Hospital of Sun Yat-sen University between March and September 2019. The mediastinoscopic lymphadenectomy procedure described here is part of the single-port inflatable mediastinoscopy procedure combined with laparoscopicassisted esophagectomy (5); thus, patients with preoperative considerations and who may have had locally advanced or suspected severe invasion were excluded. Patients with abdominal esophageal and gastroesophageal junction cancer were excluded. Patients were in a supine position (Figure 1), with single-lumen tracheal intubation and double-lung ventilation. A $3-\mathrm{cm}$ incision was made parallel to the left clavicle $2 \mathrm{~cm}$ from the supraclavicular region on the left neck (Figure 2), as previously described (5). The lymphadenectomy procedure described in this article is mainly divided into three parts, left and right RLN lymphadenectomy and subcarinal LN lymphadenectomy. Clinicopathological factors were described based on the 8th UICC TNM classification. The study was conducted in accordance with the Declaration of Helsinki (as revised in 2013). Written informed consent was obtained from the patient for publication of this study and any accompanying images.

\section{Lymphadenectomy along the left RLN}

A left neck incision was established first, and then the sternocleidomastoid muscle was pulled to the left (Figure 2, Video 1). Under direct vision, the cervical esophagus and the left RLN could be dissociated (Figure 2), as described previously (5). The LNs along the cervical RLN could be removed under direct vision (Figure $3 A$, Video 2). After the single-port inflatable surgical device was established (Figure 2B), it was filled with $10-12 \mathrm{mmHg}$ carbon dioxide (Video 1). A special upper mediastinal retractor and Maryland LigaSure are recommended in this narrow space (Figure $1 B$ ). The aortic arch was seen on the left, and the esophagus and trachea were observed on 

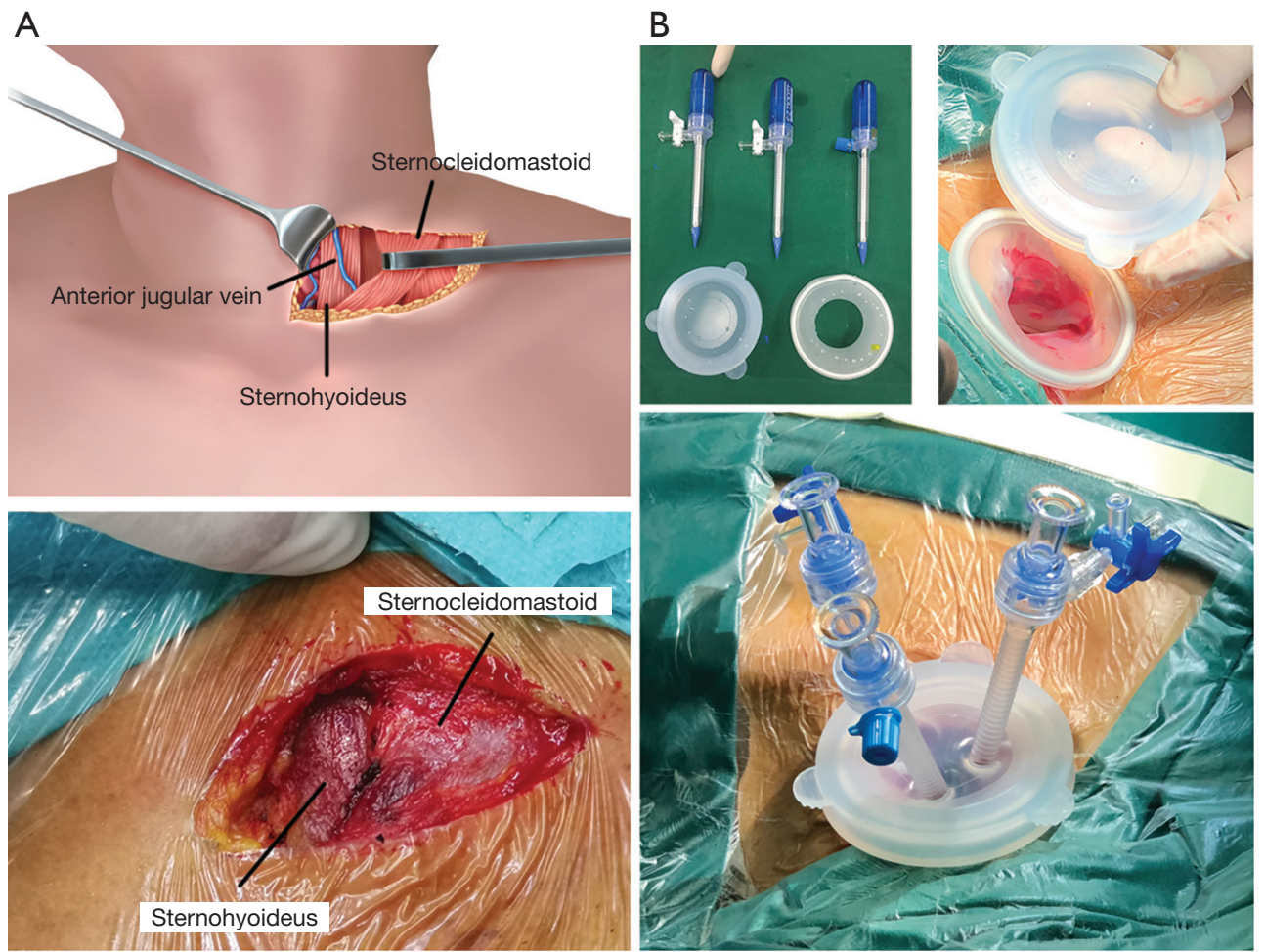

Figure 2 The establishment of trocar. (A) The incision paralleling the left clavicle at 2-cm from the supraclavicular region on the left neck. Sternothyroid and sternocleidomastoid would be pulled to the left. (B) Placement of single-port devices on the cervical incision.
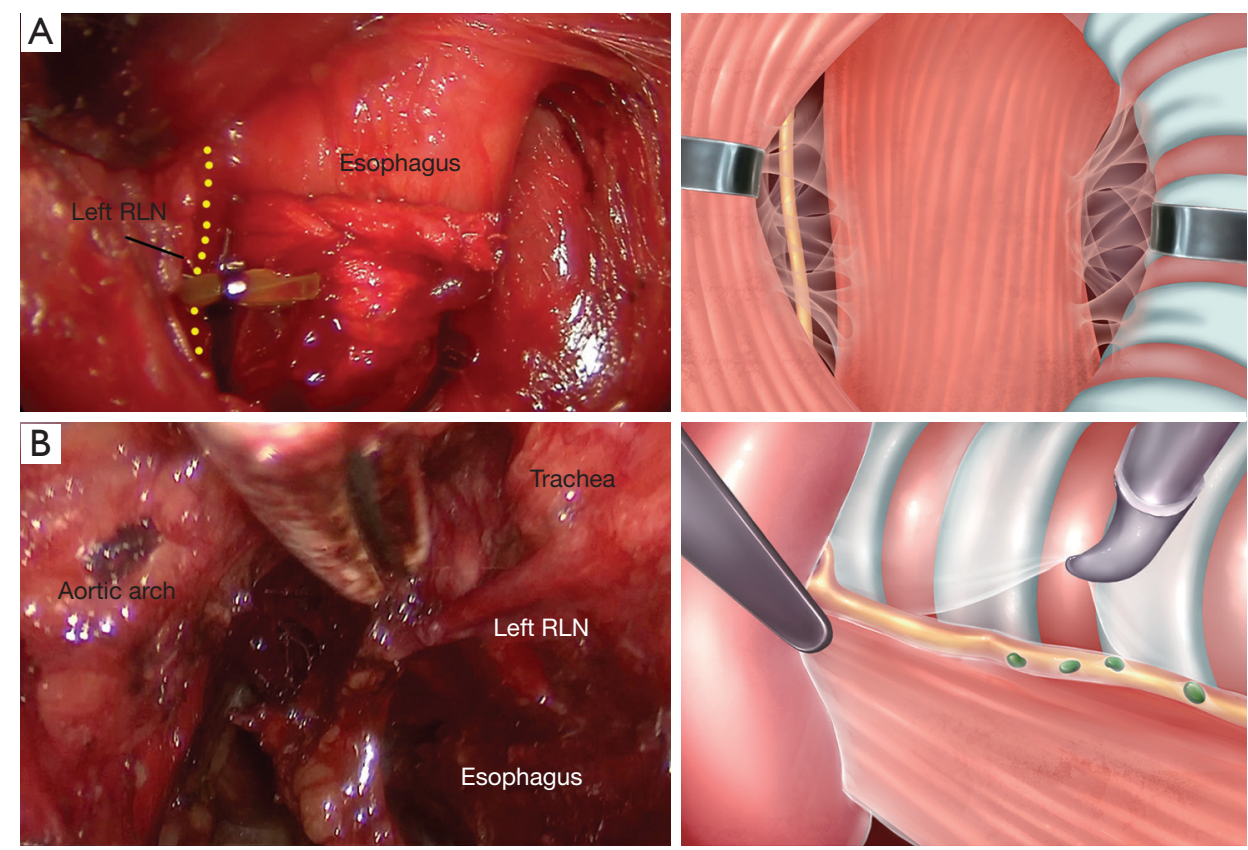

Figure 3 Lymphadenectomy along the left recurrent laryngeal nerve (A) under direct vision, the left recurrent laryngeal nerve and esophagus were marked, (B) under mediastinoscopy. The aortic arch could be seen on the left and the left recurrent laryngeal nerve and esophagus on the right. 

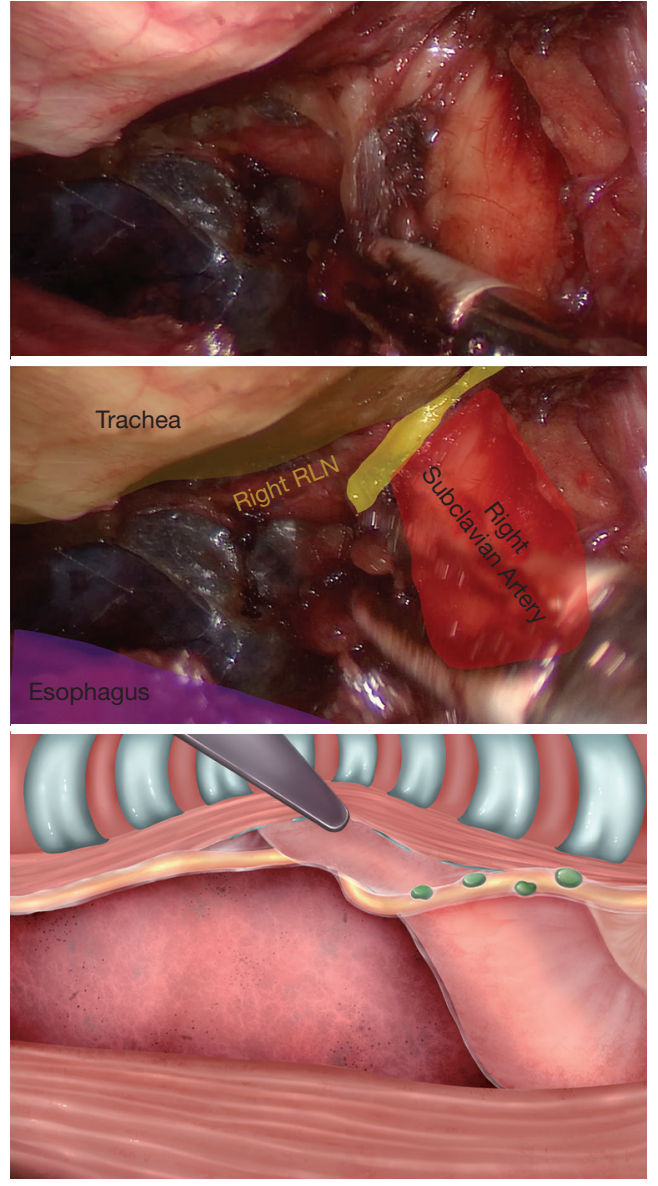

Figure 4 Lymphadenectomy along the right recurrent laryngeal nerve under mediastinoscopy. Between esophagus and trachea, the lymphadenectomy could be performed on the right upper mediastinum.

the right (Figure 3B). During dissection of the esophagus, we generally leave the left RLN on the tracheal side of the tracheoesophageal groove. Following esophageal dissociation, lymphadenectomy along the left RLN was performed from the lower edge of the thyroid (Video 3). Non-energy devices such as scissors were used, as well as energy devices. To avoid unnecessary thermal damage, we recommend using Maryland LigaSure. In addition to the possibility of causing secondary injuries, Harmonic Ace and electrocautery can generate excessive smoke during the operation, which will result in an unclear surgical field. In addition, the surface of Maryland LigaSure is relatively smooth, which can minimize damage to the surrounding tissue during the process. Starting from the lower edge of the thyroid, the LNs were swept all the way to the lower edge of the aortic arch, close to the beginning of the left RLN. To avoid damage to the tracheal membrane due to the presence of dense tissue, it is necessary to be cautious when dissociating the tissue near the tracheoesophageal groove. To prevent the LNs from being left in the mediastinal cavity, we recommend not attaching the LNs to the esophagus, but removing them en bloc.

\section{Lymphadenectomy along the right $R L N$}

After esophageal dissociation and lymphadenectomy along the left RLN, the surgical instruments could enter the right upper mediastinum between the esophagus and trachea (Figure 4, Video 4). First, the right mediastinal pleura and the right vagus nerve were revealed. LNs were removed upward along the right vagus nerve until the level of the right subclavian artery (RSA), where the RLN was observed to originate from the right vagus nerve. The surrounding LNs were dissected along the right RLN. This method was performed continuously until $2 \mathrm{~cm}$ above the upper edge of the RSA. Another method of locating the right RLN could be achieved through the RSA. When entering the right upper mediastinum, the RSA was exposed on the right side of the visual field. The RSA could then be dissected from proximal to distal. Before the right RLN has been fully exposed, it is necessary to remove the surrounding tissue and LNs around the artery and nerve.

Gentle force is required to reduce the risk of tracheal membrane injury when the trachea is lifted by the retractor during the operation. The operator should try to minimally manoeuvre energy instrument on the trailing edge of the trachea. If tracheal membrane damage occurs, conversion to open surgery needs to be carried out. Similar to the left side, we recommend removing the $\mathrm{LNs}$ en bloc.

\section{Subcarinal lymphadenectomy}

Between the trachea and esophagus, the left and right main bronchi were exposed along the trailing edge of the trachea down to the carina. Then, 106tbL/R LNs (6) were removed. Here, the carbon dioxide and the retractor were used to stretch the gap between the trachea and esophagus to form an operating space. After the lower edges of the left and right main bronchi were revealed, they were carefully freed from the outer edge along this point, and the subcarinal LNs could be easily identified (Figure $5 A$ ). The LNs were gradually detached from the trachea by the retractor and Maryland LigaSure. Careful and gentle 

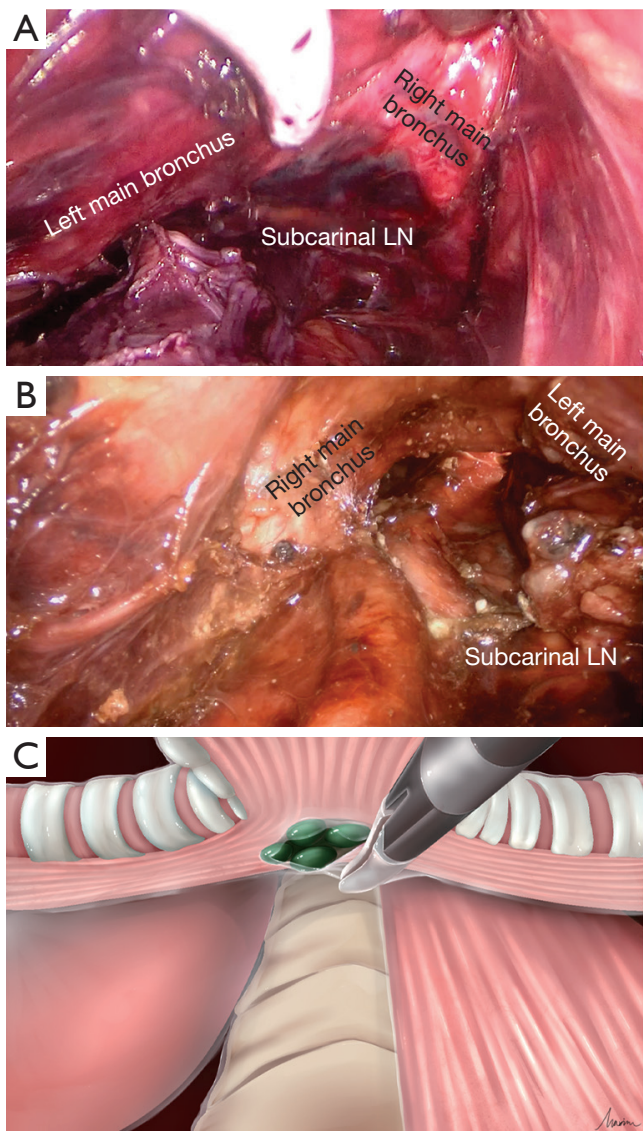

Figure 5 Subcarinal lymphadenectomy. (A) Dissection of subcarinal lymph nodes under mediastinoscopy. (B) Transhiatal subcarinal lymphadenectomy under laparoscopy. (C) The illustration of the subcarinal lymph nodes.

manoeuvring of the tracheal membrane was also required. During the operation, the tracheal membrane is always in the upper part of the visual field, so the risk of tracheal membrane injury is continuously high when removing the subcarinal LNs. Sometimes, the LNs are obscured by the carina, and there are certain difficulties during lymphadenectomy due to mediastinoscopy. However, it is not recommended for beginners to dissect the subcarinal LNs from here. The method of lymphadenectomy described in this article is part of the single-port inflatable mediastinoscopy procedure combined with laparoscopicassisted esophagectomy. Thus, it is recommended that beginners should dissect these LNs transhiatally under laparoscopy (Figure 5B), if the visual field is sufficiently clear.

Under mediastinoscopy (Video 5), dissection could take place from the left and right main bronchi along the edge of the LNs towards the carina (Figure 5C). Although the visual field is better in transhiatal $\mathrm{LN}$ resection, there are also blind spots. In addition, this approach is limited by the length of the instrument and interference with the heart during the operation. Thus, transhiatal removal of subcarinal LNs is not perfect. Because transmediastinal and transhiatal resection of subcarinal LNs have their own advantages and disadvantages, our experience is that the resection strategy should be determined on a case-by-case basis. Of these 56 patients, approximately $40 \%$ underwent mediastinoscopy, approximately $20 \%$ underwent hiatal resection, and approximately $40 \%$ underwent a combination.

\section{Comments}

We analysed 56 patients with esophageal squamous cell carcinoma from March to September 2019 who underwent this operation at the Fifth Affiliated Hospital of Sun Yat-sen University. The mean age of the 56 patients was 67.4 years, 46 were males, and 10 were females. The tumor was located in the mid-thoracic esophagus in 31 patients and in the lower thoracic esophagus in 23 patients. The preoperative TNM staging was as follows: 10 cases of T1b, 35 cases of $\mathrm{T} 2$, and 11 cases of T3; and 41 cases of N0, 12 cases of N1, and 3 cases of N2. The median number of mediastinal LNs removed was 17 (9 to 23); 6 (2 to 9) along the left RLN; and 3 ( 1 to 6 ) along the right RLN. Seven patients (12.5\%) developed RLN palsy and 5 patients (8.9\%) developed anastomotic leakage. Postoperative laryngoscopy showed that all 7 cases of RLN palsy were on the left side, and were grade 1 according to the Clavien-Dindo classification system; none of these cases were present 3 months postoperation.

Traditional non-transthoracic surgery has been achieved by mediastinoscopy-assisted transhiatal esophagectomy (7). This type of surgery has a small space and limited field of vision. With this procedure, upper mediastinal LN removal cannot be achieved, especially on the right side. The new mediastinoscopy technique proposed by Professor Fujiwara effectively avoids the disadvantages of traditional mediastinoscopy surgery. However, in this procedure, an incision on the right neck is still needed. Our single-port inflatable mediastinoscopy technology can remove the upper mediastinal LNs on both sides with a single incision on the left neck and can avoid trauma to the right neck. Moreover, the bilateral RLNs can be clearly exposed and protected under mediastinoscopy. Compared with previous 
surgical techniques, this surgery is less invasive, and the bilateral RLNs are more clearly revealed. However, the LNs can only be dissected to approximately $2 \mathrm{~cm}$ above the upper edge of the RSA along the right RLN. Therefore, this surgical method may not be suitable for all patients with esophageal cancer. The neck LNs of the patients need to be fully evaluated before surgery. This procedure is not recommended if there is a possibility of residual LNs. In the future, more experience will still be needed to further evaluate the safety and effectiveness of the procedure.

\section{Acknowledgments}

We thank Yu Ruhui (herring127@qq.com) for her great help with the illustrations in this article.

Funding: This work was supported by National Key Research and Development Program of China (2018YFC0910600).

\section{Footnote}

Conflicts of Interest: All authors have completed the ICMJE uniform disclosure form (available at: http://dx.doi. org/10.21037/tcr-20-467). The authors have no conflicts of interest to declare.

Ethical Statement: The authors are accountable for all aspects of the work in ensuring that questions related to the accuracy or integrity of any part of the work are appropriately investigated and resolved. The study was conducted in accordance with the Declaration of Helsinki (as revised in 2013). Written informed consent was obtained from the patient for publication of this study and any accompanying images.

Cite this article as: Gan X, Zhong H, Li X, Wang X, Huo W, Wu T, Zhong B, Zhang B, Cheng H, Wu X, Cao Q. Mediastinal lymphadenectomy under laparoscopic assisted single-port inflatable mediastinoscopy through left neck approach. Transl Cancer Res 2020;9(8):4976-4981. doi: 10.21037/ tcr-20-467
Open Access Statement: This is an Open Access article distributed in accordance with the Creative Commons Attribution-NonCommercial-NoDerivs 4.0 International License (CC BY-NC-ND 4.0), which permits the noncommercial replication and distribution of the article with the strict proviso that no changes or edits are made and the original work is properly cited (including links to both the formal publication through the relevant DOI and the license). See: https://creativecommons.org/licenses/by-nc-nd/4.0/.

\section{References}

1. Bray F, Ferlay J, Soerjomataram I, et al. Global cancer statistics 2018: GLOBOCAN estimates of incidence and mortality worldwide for 36 cancers in 185 countries. CA Cancer J Clin 2018;68:394-424.

2. Feng RM, Zong YN, Cao SM, et al. Current cancer situation in China: good or bad news from the 2018 Global Cancer Statistics? Cancer Commun (Lond) 2019;39:22.

3. Canet J, Gallart L, Gomar C, et al. Prediction of postoperative pulmonary complications in a populationbased surgical cohort. Anesthesiology 2010;113:1338-50.

4. Fujiwara H, Shiozaki A, Konishi H, et al. SinglePort Mediastinoscopic Lymphadenectomy Along the Left Recurrent Laryngeal Nerve. Ann Thorac Surg 2015;100:1115-7.

5. Li Z, Li Y, Wang L, et al. Management of calcified hilar lymph nodes during thoracoscopic lobectomies: avoidance of conversions. J Thorac Dis 2019;11:657-63.

6. Japan Esophageal Society. Japanese Classification of Esophageal Cancer, 11th Edition: part I. Esophagus 2017;14:1-36.

7. Fujiwara H, Shiozaki A, Konishi H, et al. Mediastinoscope and laparoscope-assisted esophagectomy. J Vis Surg 2016;2:125. 